\title{
PSICOLOGÍA CIENTÍFICA Y PRÁCTICA PROFESIONAL DEL PSICÓLOGO: DOS CARAS DE UNA MISMA MONEDA
}

Manuel de Vega

Universidad de La Laguna

\section{RESUMEN}

Se argumenta que el auge de la psicologia en nuestra sociedad se explica por el desarrollo de la psicología científica. El psicólogo profesional debe tener una formación científica, pero además debe tener conocimientos de análisis de tareas, evaluación de resultados, combinar conocimientos declarativos y procedimentales, $y$ saber aprender. El profesor de psicología debe compartir estos conocimientos y destrezas, pero además debe saber investigar, enseñar, debe socializarse cientfficamente, asumir la cultura de la evaluación, y saber ser productivo en un medio institucional muy lejano de la idoneidad.

Palabras clave: CONOCIMIENTO DECLARATIVO Y PROCEDIMENTAL, SABER APRENDER, PSICOLOGIA CIENTIFICA, ANÁLISIS DE TAREAS, EVALUACIÓN DE RESULTADOS, SOCIALIZACIÓN DEL CIENTIFICO, CULTURA DELAEVALUACIÓN, INSTTTUCIÓNACADÉMICA. 


\section{SUMMARY}

The growing influence of psychology in our society can be partially explained by the development of scientific psychology. The professional psychologist must be scientifically trained. $\mathrm{He} / \mathrm{she}$ must be also skillful in tasks analysis, evaluation of results, balanced in his/her declarative and procedural knowledge, and capable to leam. The teacher of psychology shares these knowledge and skills, but also must be able to research, to teach, to get socialized in the scientific community, to assume the evaluation culture, and to know how to be productive in a non-optimal institutional environment.

Key words: DECLARATVE AND PROCEDURAL KNOWLEDGE, KNOWING HOW TO LEARN, SCIENTIFIC PSYCHOLOGY, TASKS ANALYSIS, RESULTS EVALUATION, SCIENTIST SOCIALIZATION, EVALUATION CULTURE, ACADEMIC INSTTUTIONS.

Si observamos la evolución histórica de la profesión de psicólogo podemos asegurar sin pecar de exceso de optimismo que ésta ha tenido un éxito notable. De ser prácticamente inexistente hace apenas un siglo, la psicología ha pasado a ser una profesión dinámica y completamente enraizada en nuestra sociedad. Los profesionales de la psicología han ido ampliando su campo, ofreciendo servicios cada vez más diversos a la sociedad. Por poner algunos ejemplos, los psicólogos diagnostican y tratan' trastornos emocionales; miden las actitudes intelectuales; evalúan la personalidad normal o psicopatológica; describen los déficits cognitivos de los pacientes cerebrales; tratan a las personas con autismo; prestan ayuda a los pacientes oncológicos; contribuyen a paliar el deterioro cognitivo de los pacientes de Alzheimer; diagnostican y tratan problemas de aprendizaje en los niños; evalúan el perfil psicológico y profesional

1. En este artículo utilizaré de forma algo lasa téminos como "diagnóstico", "Iratamiento", "evaluación" en lugar de otros quizá más precisos pero también más aparatosos como "psicodiagnóstico", "intervención psicológica" o "evaluación psicológica". 
de los candidatos a un puesto de trabajo; atienden a las víctimas de catástrofes, del terrorismo, de violencia doméstica, de abuso sexual, o de acoso laboral; contribuyen a la mejora del rendimiento y de la satisfacción laboral; evalúan testimonios en contextos jurídicos; tratan los problemas del adolescente (conductas de riesgo, trastomos en la ingesta, etc.) e, incluso, asesoran a los astronautas para facilitar su adaptación mental e interpersonal en el espacio.

¿Por qué este auge de la profesión del psicólogo? La respuesta puede parecer obvia: la psicología es una disciplina aplicada que como acabamos de ver- contribuye a aminorar el sufrimiento y a mejorar la calidad de vida de la gente. Por tanto, los psicólogos tienen un amplio espacio de problemas prácticos para desarrollar su profesión. Sin embargo, este argumento es un tanto circular, pues es como asegurar que los bomberos son necesarios porque existen los incendios, o los médicos lo son porque existen las enfermedades.

En realidad, no creo que la existencia de problemas humanos por si sola determine la consolidación de una profesión, sino que hace falta algo más. Por ejemplo, la profesión médica en el mundo medieval era una actividad bastante marginal, mezcla de supersticiones, tradiciones, y conocimientos empíricos derivados de la práctica. El médico sólo alcanzaría gradualmente el estatus profesional y el prestigio social que goza en la actualidad a medida que la medicina científica fue acumulando conocimientos (v.g., anatomía y fisiología, el descubrimiento de los gérmenes y de los virus como agentes de la enfermedad, las bases genéticas de la herencia y de algunas patologías, etc.). Esa misma medicina ciertífica permitio, además, desarrollar técnicas eficaces (instrumentos de diagnóstico, vacunas, antibióticos, procedimientos quirúrgicos, etc.), de modo que cuando el moderno médico profesional se enfrenta a la práctica tiene a su alcance un inmenso repertorio de conocimientos sistematizados (tanto teóricos como prácticos) y de técnicas de diagnóstico y tratamiento de eficacia contrastada.

De forma análoga, el auge actual de la profesión de psicólogo es paralelo al desarrollo de la psicología científica. Cuando Wundt creó el primer laboratorio de psicología experimental en Alemania o, unos años más tarde, Binet diseñó en Francia el primer test de inteligencia, se sentaron las bases de una nueva y pujante disciplina 
académica. La psicologla científica se asentó firmemente en el método experimental, y construyó una sofisticada teoria de la medida para estudiar los fenómenos mentales y de la conducta. Esto fue muy revolucionario en su momento, pues supuso una ruptura con la tradición humanista secular que afrontaba el estudio de la mente utilizando procedimientos cualitativos como la introspección y la argumentación filosófica. Los métodos cualitativos -con la notable excepción del psicoanálisis- apenas impulsaron el desarrollo de la psicología como profesión. Han sido las teorias científicas del aprendizaje, de la inteligencia, de los procesos cognitivos, o de la personalidad, surgidas de los laboratorios, de la investigación psicométrica y de los estudios de campo, las que han permitido generar técnicas eficaces aplicables en el mundo profesional. Así, se crearon diversos tests para medir la inteligencia, la personalidad, y otros rasgos psicológicos; se diseñaron técnicas conductuales y cognitivas para el tratamiento de trastornos psicológicos; se aplicaron programas de intervención comunitaria para mejorar el rendimiento, resolver conflictos grupales, o contribuir al desarrollo humano, etc.

\section{SABER PSICOLOGÍA CIENTÍFICA}

A partir de la breve reflexión histórica que acabamos de hacer ya tenemos una respuesta provisional a la pregunta ¿qué debe saber el psicólogo?. El psicólogo debe tener una formación científica sólida que avale su actividad profesional. Esta primera respuesta no parece muy reveladora ni polémica. Muy pocos psicólogos la pondrían en duda hoy en día. De hecho, los curricula de psicología en las universidades españolas, y en la mayoria de los países de nuestro entorno, incluyen los contenidos y métodos propios de la psicología científica y las técnicas derivadas de ella. Sin embargo, no está de más insistir en ello porque la Psicología, al igual que le ocurre a la Medicina, sufre un considerable acoso por parte de las pseudociencias. Hay diversas formas de "psicologias alternativas" que, utilizando un lenguaje pseudocientífico, pretenden ofrecer soluciones no convencionales, que no han sido contrastadas científicamente. El buen profesional de la psicología, en cambio, no dispone de soluciones 
mágicas, sino que se basa en conocimientos que han sido fruto del trabajo riguroso y paciente de generaciones de investigadores, y de profesionales. Estos conocimientos científicos son una garantía del éxito, tanto individual como colectivo, y distinguen a los psicólogos de los simples aficionados.

Hay algunas razones por las que la profesión de psicólogo es especialmente vulnerable al intrusismo de individuos con escasa 0 nula formación científica. Una de ellas es que todos los seres humanos compartimos unas notables capacidades mentalistas, de modo que cualquier charlatán puede erigirse en "psicólogo" basándose en sus intuiciones y reflexiones ingenuas. Todos disponemos, en efecto, de una "teoría de la mente", como parte del equipo cognitivo básico de nuestra especie. Esta teoría de la mente nos permite entender, predecir y hasta manipular los estados mentales de los demás. Por ejemplo, inferimos con bastante precisión las intenciones, emociones o metas de las personas con las que nos relacionamos. Por otra parte, somos capaces de distinguir entre las creencias o conocimientos de los demás y las nuestras propias. Por ejemplo, durante una conversación con un amigo, "sé lo que yo sé", pero también "sé -en alguna medida- lo que el otro sabe" y ello me permite seleccionar pragmáticamente qué debo decir y que debo callar.

Estas poderosas capacidades mentalistas nos hacen humanos y nos alejan considerablemente de nuestros parientes primates. Además son fenómenos psicológicos fascinantes que han sido objeto ellas mismas de investigación científica por parte de los psicólogos evolutivos, como Alan Leslie, psicólogos del razonamiento como Leda Cosmides, o psicólogos comparados como David Premack. Sin embargo, más allá de su ámbito natural de aplicación estas habilidades pragmáticas no permiten construir conocimientos psicológicos rigurosos y útiles para el profesional. Lo más probable es que una teoría psicológica basada únicamente en las intuiciones mentalistas sea profundamente errónea. Como asegura Vicente Pelechano, refiriéndose a lo engañoso de las formulaciones simplistas: "lo fácil no ha funcionado demasiado bien" en psicología. Lo obvio, lo aparentemente sensato, lo que todo el mundo acepta sin gran discusión puede ser simplemente falso. Muchos procesos mentales son simplemente inaccesibles a la conciencia y, por tanto, 
no pueden analizarse intuitivamente. Por ejemplo, nuestra intuición no nos dice nada sobre cómo funcionan los procesos de lenguaje (cómo reconocemos las palabras, aplicamos principios gramaticales en la construcción de frases, etc.), ni como operan los mecanismos de la memoria (cómo recordamos, cómo olvidamos, cómo se organiza la información en la memoria). Ni siquiera en el terreno interpersonal -más próximo a la teoría de la mente- disponemos de intuiciones fiables sobre las causas remotas del comportamiento, de las emociones, de los afectos, o de los estados de ansiedad, como el propio Sigmund Freud señaló acertadamente.

Por otra parte, el razonamiento "natural" o pragmático tan útil en la resolución de problemas cotidianos, implica ciertos sesgos y distorsiones que nos pueden conducir a hipótesis y teorías psicológicas poco realistas. Uno de estos sesgos es suponer que hay cierta analogía entre las causas y los efectos. Esto ha llevado a postular teorías falsas y prácticas psicológicas erróneas e, incluso, perjudiciales. Por ejemplo, durante años la dislexia se consideró como un problema perceptivo, ya que los disléxicos parecían confundir palabras de apariencia similar, o bien letras basadas en el mismo patrón visual invertido, como " $b$ " y "d", o " $p$ " y "q". En consecuencia se prescriblan al disléxico ejercicios de entrenamiento visual o, incluso, psicomotor para superar su problema. Hoy sabemos, gracias a las investigaciones de los psicolingüístas como Charles Perfetti, que el problema del disléxico se manifiesta en la lectura pero es, en realidad, un déficit general del lenguaje localizado en el procesamiento de fonemas, o en la representación de las palabras jen el habla!. El razonamiento por analogía (confunde letras, por tanto tiene un problema de reconocimiento visual) era intuitivamente impecable... pero falso.

Sólo los conocimientos científicos le van a proporcionar al psicólogo profesional criterios para separar el grano de la paja, en medio de la multitud de falsas teorlas, intuiciones injustificadas, y simple palabrería que amenazan la práctica profesional. Los conocimientos científicos también le permitirán conocer la base teórica de los instrumentos que utiliza, evitando una aplicación meramente mecánica y rutinaria. Por ejemplo, podrá entender la teoría de la personalidad que hay detrás de la aplicación de un test que mide la introversión, 
- comprender que los test que miden aptitudes se basan en una concepción "modular" de la inteligencia, es decir, como un conjunto de factores independientes.

Pero, la formación científica no consiste en mera erudición. No se trata únicamente de que el psicólogo adquiera una serie de conocimientos estáticos o inertes, a veces dilíciles de conectar con los problemas del mundo real. El psicólogo profesional debe, además, disponer de un conjunto de habilidades procedimentales que se derivan de su formación científica. Por ejemplo, la sensibilidad cuantitativa en los procesos de evaluación, diagnóstico e intervención, la capacidad de búsqueda y asimilación de nueva información, la capacidad para diseñar tareas y planificar su intervención profesional, o las destrezas para trabajar en equipo. Voy a tratar de sintetizar algunas de estas capacidades en los siguientes puntos: a) saber analizar tareas; b) saber evaluar los resultados de su intervención; c) saber combinar la palabra y la acción; d) saber aprender.

\section{SABER ANALIZAR TAREAS}

Angel Rivière solía decir que un psicólogo es un experto en análisis de tareas. Esto puede parecer una simplificación excesiva ya que, con el mismo grado de adecuación, podríamos asegurar según nuestras preferencias académicas- que el psicólogo debe ser un experto en análisis de la conducta, en análisis de los procesos cognitivos, en análisis de los rasgos de personalidad, en análisis interpersonal, o en análisis de interacciones entre grupos. Cualquiera de estas opciones tiene sus propias técnicas y métodos; sin embargo, todas ellas comparten el principio general del análisis de tareas. Esto es, parten de un diseño sistemático de un conjunto de situaciones bien definidas, lo cual incluye: a) estímulos discretos estandarizados, b) ordenación o programación temporal de estos estímulos, c) registro de respuestas del individuo ante estos estímulos, d) posterior análisis cuantitativo de resultados. El análisis de tareas es obvio en el caso de un planteamiento conductista. En desarrollo de un programa de modificación de conducta, por ejemplo, supone un contexto de tareas cuidadosamente definidas, incluyendo 
la planificación de los estímulos, de las contingencias de refuerzo y el registro de las respuestas del individuo. Respecto a los planteamientos cognitivos, aun cuando los procesos que se pretende estudiar o modificar no sean observables en sí mismos (expectativas, creencias, valores, etc.), el psicólogo cognitivo no difiere mucho del conductista en la necesidad de diseñar situaciones bien controladas, elaborar estímulos, y registrar sistemáticamente respuestas; es decir, de nuevo análisis de tareas. Lo que añade el psicólogo cognitivo es el establecer una relación entre las micro-tareas diseñadas y los componentes mentales que subyacen a su realización.

El análisis de tareas es además una tecnología psicológica exportable a otras disciplinas. Así las modernas técnicas de neuroimagen sólo permiten desvelar las estructuras cerebrales relacionadas con una determinada actividad mental, utilizando métodos sustractivos, es decir, restando el registro cerebral de los participantes mientras realizan dos tareas diferentes cuidadosamente diseñadas. Por ejemplo, para desvelar qué estructuras tienen relación con el acceso léxico, se puede registrar la actividad cerebral en dos condiciones de tarea: la lectura de palabras y la lectura de pseudopalabras. Ambas tareas son similares en muchos aspectos aunque se diferencian en un aspecto crítico: el significado. Al restar las imágenes cerebrales correspondientes a ambas tareas se obtendrá un registro de estructuras neuronales específicas del reconocimiento de las palabras.

Una observación cautelar: los tests y cuestionarios estandarizados que se encuentran a disposición del psicólogo profesional constituyen una poderosa herramienta, pero su uso indebido podría llevarle a prescindir del análisis de tareas. Esto puede resultar paradójico ya que, por sí mismos, los tests son el exponente de una tecnología muy refinada de análisis de tareas (análisis de items, factorización, baremación, medidas de fiabilidad y validez). Sin embargo, el usuario del test puede limitarse a aplicar y corregir el test según las prescripciones del manual, lo cual es útil como instrumentación auxiliar, pero francamente rutinario si sólo se hace eso. El conocimiento del análisis de tareas que propugno aquí, es un conocimiento mucho más flexible y generativo, y también más esforzado; requiere que el psicólogo sepa programar y diseñar tareas a la medida de cada 
situación de intervención. Por ejemplo, debe ser capaz de diseñar un protocolo de análisis y registro de conductas para el tratamiento de un riño autista, o construir la jerarquía de situaciones en el tratamiento de una fobia por desensibilización sistemática, o diseñar un programa de intervención comunitaria. Ello no significa en modo alguno prescindir de los análisis de tarea "prefabricados" como son los tests y cuestionarios, de enorme utilidad en tareas de evaluación y diagnósticas. Pero en la faceta de intervención, el profesional debe saber algo más: diseñar sus propias tareas.

\section{SABER EVALUAR RESULTADOS}

El énfasis en la evaluación de resultados debe ser una guía constante en la actividad profesional. El psicólogo no sólo debe evaluar resultados, sino que debe saber hacerlo de forma cuantitativa. En este sentido, una ventaja más del análisis de tareas es facilitar al psicólogo la evaluación de los resultados de su investigación o intervención, ya que el análisis de tareas implica un registro operacional de respuestas. Por otra parte, algunas técnicas de intervención como las terapias cognitivo-conductuales incluyen en su diseño y aplicación el registro y la evaluación cuantitativa de resultados.

En el contexto profesional podría parecernos que la evaluación cuantitativa de resultados es innecesaria o poco práctica, más propia de la investigación de laboratorio que del "mundo real". Algunos psicólogos podrían concluir que consume un tiempo precioso que podría dedicarse a otros problemas más urgentes, por ejemplo a tratar nuevos pacientes. Sin embargo, la evaluación cuantitativa de resultados tiene ventajas evidentes con respecto a las meras impresiones subjetivas del psicólogo o de los receptores del tratamiento psicológico. Consideremos el caso de la evaluación de resultados en las psicoterapias analíticas. La ausencia de operacionalización conduce al puro subjetivismo. Es decir, que no hay criterios objetivos que puedan determinar el fin o la continuación de la terapia, es casi imposible contrastar la eficacia de tratamientos alternativos, no hay medidas de acuerdo entre observadores, etc. 
La evaluación cuantitativa de resultados, en cambio, permite contrastes intra-sujeto, o intra-grupo de los efectos de una intervención (contraste "antes y después"). También se pueden contrastar los resultados con normas externas, criterios o baremos. Todo esto permite no sólo una mejor evaluación del receptor o paciente, sino también de la eficacia de la propia técnica de intervención en comparación con otras alternativas.

\section{SABER COMBINAR LA PALABRA Y LA ACCIÓN}

Algunos psicólogos, como John R. Anderson, suelen distinguir dos tipos de conocimientos generales: los declarativos y los procedimentales. Los conocimientos declarativos ("saber qué") son explícitos o conscientes, y se expresan (o "declaran") verbalmente. Por ejemplo, son declarativos el conocimiento que recuperamos cuando contestamos a las preguntas de un examen, o el conocimiento que utilizamos para contar episodios autobiográficos. En cambio los conocimientos procedimentales ("saber cómo") son destrezas o habilidades, tienen un carácter implícito, es decir, son difíciles de verbalizar, y se expresan en la acción. En muchas actividades humanas se combinan ambos tipos de conocimiento. Por ejemplo, para conducir coches debemos aprender el código de la circulación (declarativo), pero también a conducir realmente por la ciudad o la carretera (procedimental). Ambos tipos de conocimiento por sí mismos son insuficientes para adquirir un dominio de la conducción. El recitado perfecto del código de la circulación no le capacita a uno ni siquiera para poner en marcha el coche. Pero un aprendizaje exclusivamente procedimental de la conducción sería poco adaptativo y hasta peligroso, al no poder interpretar el individuo el conjunto de instrucciones (prohibiciones, obligaciones 0 recomendaciones) que debe seguir continuamente. Lo ideal es una buena integración entre ambos tipos de conocimiento.

En la profesión de psicólogo ocurre exactamente lo mismo. Deben aplicarse de forma equilibrada conocimientos declarativos y procedimentales. No siempre se ha conseguido ese equilibrio, y en la práctica profesional nos podemos encontrar con excesos en uno 
u otro sentido. Desde profesionales que se basan casi exclusivamente en la palabra (excesos declarativos), hasta aquellos que aplicación rutinaria e irreflexiva técnicas o procedimientos estandarizados (excesos procedimentales). En el polo de los excesos declarativos hemos de mencionar las clásicas terapias psicodinámicas, en las que el paciente y el psicoterapeuta desarrollan una interacción verbal a lo largo de un buen número de sesiones. Un objetivo de esta interacción suele ser que el terapeuta - o el propio paciente con su ayudaconsiga descubrir e interpretar los motivos ocultos de sus traumas o neurosis. Las terapias psicodinámicas se basan en la creencia de que hacer explícitos o "declarar" las conflictos y las causas subyacentes de los conflictos permite superarlos. Sin embargo, es una creencia, como mínimo, algo exagerada. Es posible que una terapia "declarativa" pueda producir un alivio transitorio en un paciente, por ejemplo aquejado de angustia o estados depresivos. Pero, es dudoso que produzca cambios permanentes en sus hábitos, creencias y emociones. Los estudios clásicos de Hans Eysenck sobre la escasa eficacia del psicoanálisis así parecen mostrarlo.

Los excesos interpretativos o declarativos pueden ser inadecuados pero, en el polo opuesto también puede haber excesos procedimentales. Es el caso del psicólogo que se convierte en mero tecnólogo, por ejemplo, aplicando tests mecánicamente cual si se tratase de una actividad burocrática; o aplicando una técnica conductual de modo análogo a quien aplica un condicionamiento operante a una rata. Después de todo el psicólogo trabaja con seres humanos y les debe una explicación sobre su estrategia de intervención, el posible origen del problema, los progresos que cabe esperar, o los resultados que se están obteniendo.

\section{SABER APRENDER}

Imaginemos a una joven licenciada que acaba de estrenar su flamante título de Psicología. La nueva licenciada ha sido una estudiante excepcional, ha cursado un plan de estudios inmejorable, $y$ ha tenido la suerte de encontrarse con profesores competentes y motivados. Siguiendo su buena racha, la psicóloga es 
contratada inmediatamente en un centro de psicología infantil, donde se enfrenta por primera vez a un trabajo real. A su despacho, llegarán niños con todo tipo de problemas: dislexia, hiperactividad, trastornos de ansiedad, autismo, etc. ¿Estará en condiciones de afrontar la evaluación y tratamiento de estos problemas? La respuesta es sí y no. Su buena formación académica le garantiza unos conocimientos declarativos sobre las categorías básicas de problemas infantiles. También dispondrá, al salir de la universidad, de conocimientos procedimentales sobre la entrevista diagnóstica, la exploración y evaluación del niño, o el diseño de programas de intervención. Sin embargo, es fácil predecir que a la nueva profesional le faltará algo. Por supuesto, le faltará eso que solemos llamar "experiencia". Pero la experiencia se adquiere con el tiempo, cuando se cuenta con una formación inicial y una motivación adecuadas. De modo que 263 años después nuestra psicóloga tendría sin duda esa experiencia. Pero aún le faltaría algo más... seguir aprendiendo.

Creo que es un error dar por clausurada la formación al terminar los estudios de licenciatura. Como ocurre con los profesionales de la medicina, el psicólogo debe mantener una capacidad de aprendizaje o de formación continua a lo largo de su carrera profesional. El dinamismo de la profesión de psicólogo hace imprescindible esa actitud de aprendizaje continuo. Nuestra profesión, como ya hemos señalado, responde a nuevos desafíos y tiende a ampliar su ámbito de aplicación. Los avances científicos ofrecen periódicamente una mejor comprensión de los viejos problemas, los desarrollos profesionales mejoran las técnicas de evaluación y los procedimientos de intervención, etc. El psicólogo debe estar abierto a este flujo de información profesional, y no encasillarse en su nivel de formación inicial. Hay varios modos de actualizarse o mantenerse al día. Participando en cursos y seminarios de post-grado dirigidos a la especialización o ampliación de conocimientos; manteniendo hábitos de lectura de revistas profesionales y científicas de su especialidad; o acudiendo a congresos y reuniones profesionales para intercambiar conocimientos y experiencia con otros colegas. A nuestra hipotética psicóloga recién licenciada habria que decirle que practique intensamente su profesión y... que siga estudiando. 
El entorno laboral del profesor de psicología es muy diferente del psicólogo profesional (aun cuando a veces el profesor alterna su actividad académica con la profesional). Todo lo que he mencionado hasta ahora sobre lo que debe saber el psicólogo, es aplicable también al profesor aunque, sin duda, con énfasis algo diferentes. El profesor debe tener unos conocimientos científicos aún más sólidos que el profesional, debe saber hacer análisis de tareas especialmente en contextos de investigación, debe saber evaluar cuantitativamente resultados aplicando elaboradas técnicas estadísticas, mantener un equilibrio entre conocimientos declarativos y procedimentales tanto en la docencia como en la investigación y, por supuesto, debe saber aprender.

Sin embargo, el profesor afronta desafíos propios del ámbito académico que requieren conocimientos y destrezas específicos. Voy a destacar algunos: a) debe saber investigar; b) debe saber enseñar; c) debe socializarse en ámbitos científicos; c) debe asumir la cultura de la evaluación; d) saber adaptarse a un medio institucional poco idóneo.

\section{SABER INVESTIGAR}

Las dos funciones fundamentales del profesor universitario, como ya destacaba Ortega y Gasset, son investigar y enseñar. Con frecuencia en nuestro país la opinión pública, los dirigente políticos e, incluso, los profesores universitarios se limitan a valorar la docencia como la única actividad socialmente relevante. La investigación pasa a un segundo plano y se considera, en todo caso, como una actividad un tanto elitista que sólo beneficia a quien la practica, satisfaciendo su vocación o sus ambiciones personales. Naturalmente, esta visión del mundo académico es profundamente errónea y socialmente miope, y lo es especialmente en psicologia en donde, como hemos señalado, la formación científica subyace a la misma práctica profesional.

El profesor debe dedicar un porcentaje importante de su actividad profesional a investigar. Ello supone, en primer lugar, saber trabajar de modo cooperativo con otras personas, es decir, dirigir o integrarse 
en proyectos de investigación con otros colegas. Además, requiere aprender a emplear técnicas y procedimientos experimentales, psicométricos u observacionales, dependiendo de la naturaleza de su trabajo; dominar el uso de los estadísticos adecuados; impartir cursos de doctorado; dirigir tesis doctorales; hacer búsquedas bibliográficas eficientes; leer y asimilar con facilidad la información publicada sobre su campo; redactar informes sobre los resultados de su investigación, etc.

Las destrezas del investigador requieren un "formateado" temprano de la mente, pues son difíciles de adquirir en etapas más tardías. Es necesario, pues, que el joven investigador se integre desde el comienzo en entornos institucionales favorables. Por ejemplo, juegan un papel muy relevante en los primeros años de la carrera académica, los primeros maestros en sentido académico, muy especialmente el director de la tesis doctoral, la disponibilidad de recursos y tiempo suficiente, y la valoración social de su actividad. El profesor que no haya tenido estas oportunidades iniciales, aun cuando pueda ser un buen docente, tendrá dificultades para incorporarse a líneas de investigación productivas. Es probable que se refugie en la erudición, es decir, que acumule conocimientos sobre lo que han dicho y hecho otros, pero sin aportar su grano de arena al conocimiento. De nuevo se nos plantea la dicotomía entre saber declarativo y el saber procedimental. El aspecto procedimental de la actividad científica es "saber investigar", y este tipo de saber debe estar incorporado al perfil básico del profesor de psicología.

\section{SABER ENSEÑAR}

Como decía Freud enseñar es una de esas tareas imposibles, al igual que gobernar y curar. $Y$ aún más imposible es tratar de hacer explícitas las destrezas que determinan que un profesor sea bueno (jaunque quizá lo más imposible de todo enseñar a enseñar!). Y sin embargo, la diferencia entre un buen profesor universitario y uno mediocre es enorme. Los alumnos perciben al primero como un privilegio y al segundo como un castigo. Todos podemos acudir a nuestros recuerdos biográficos para recrear las clases de aquel 
profesor que despertó nuestra curiosidad, alimentó nuestro interés y nos hizo disfrutar del proceso de aprendizaje. También podemos recordar el tormento de las clases de ese otro profesor incompetente que destruía la magia del conocimiento, convirtiendo el diamante en carbón.

Es fácil establecer ciertos indicadores externos que suelen estar asociados a la docencia de calidad. Un buen indicador en general es el grado de satisfacción de los alumnos, siempre que la medición de esta satisfacción cumpla unas garantias técnicas (obtenida a lo largo de varios cursos, con una muestra representativa, preguntas representativas, etc.) Otros indicadores objetivos son la puntualidad, el establecimiento y cumplimiento de los contenidos y normas de la asignatura, el cumplimiento de las horas de tutoría, la publicación de manuales de calidad, el uso de medios didácticos (transparencias, Power Point, páginas web), etc. Otras facetas de la docencia universitaria son mucho más sutiles y difíciles de establecer: la claridad expositiva, la estimulación de la curiosidad y reflexión del alumno, la vinculación entre la teoría y la práctica, etc.

Quiero insistir en que docencia e investigación no son habilidades contrapuestas en el profesor universitario. No comparto los planteamientos maniqueos de los que aseguran que el profesor debe ser un buen docente más que un investigador, o los que aseguran lo contrario. Probablemente, investigar y enseñar sean capacidades ortogonales $u$ independientes. Mi impresión, aunque no dispongo de datos específicos, es que hay profesores que enseñan bien e investigan bien, otros que enseñan bien e investigan mal (o no investigan), los hay que enseñan mal e investigan bien $y$, finalmente, los que enseñan mal e investigan mal (o no investigan). Sin embargo, es importante destacar que un profesor que investigue bien no tiene porque ser un mal docente. Por otra parte, creo que un profesor que además de dar clases investigue puede trasmitir al alumno algo especial: una actitud merital inquisitiva que lleva a la búsqueda de conocimiento, y una confianza epistémica en que podemos contribuir a ese conocimiento. Por el contrario, un profesor que jamás investiga puede inducir a sus alumnos a pensar que el conocimiento está ya cerrado, o bien que los protagonistas de su avance son siempre "otros", generalmente con apellido anglosajón. 


\section{SOCIALIZARSE EN ÁMBITOS CIENTÍFICOS}

La ciencia es una actividad profundamente extravertida y cosmopolita. La imagen del sabio aislado en una torre de marfil y que guarda celosamente sus conocimientos esotéricos, es propia de la edad media. La imagen del científico moderno es la de un personaje comunicativo que interactúa afanosamente con otros científicos, e intenta hacer públicos lo antes posible sus descubrimientos. El propio Galileo, que algunos consideran el primer científico moderno, escribió sus obras en italiano, en lugar del latín, para que pudiese leerla más gente, y se carteaba con todos los científicos europeos de su época, influyéndoles y dejándose influir por ellos. El profesor de psicología debe incorporar estos hábitos "extravertidos" propios de la ciencia. Esto supone romper con inercias locales y costumbres endogámicas, a veces fuertemente arraigadas. Por ejemplo, debe incrementar su movilidad asistiendo a congresos de su especialidad, - visitando a colegas de otras universidades. Estos viajes son esenciales para conocer a otros investigadores, intercambiar información específica, aprender nuevas técnicas, diseñar investigaciones conjuntas, planificar intercambios de alumnos y profesores (programa Sócrates), escribir artículos en colaboración, etc. Afortunadamente, los intercambios se ven favorecidos hoy en día gracias a la difusión masiva de información por internet y al correo electrónico. También hay que mencionar la pertenencia a asociaciones especializadas, la dirección de revistas, la pertenencia a comités editoriales, la colaboración en la revisión de artículos, etc.

Otro aspecto de la socialización consiste en utilizar canales de publicación internacional (sin descuidar las revistas nacionales de calidad). El trabajo científico de un psicólogo, por valioso que sea, puede permanecer oculto o poco visible a la comunidad internacional si no se difunde en revistas de amplio impacto. Es importante que el investigador, además de hacer buena investigación, adquiera la habilidad de escribir artículos publicables. Se trata de una técnica que generalmente no se enseñan explícitamente. Por ejemplo, cómo dimensionar adecuadamente un artículo, cómo adquirir el estilo escueto de la comunicación científica (eliminando redundancias, elementos retóricos innecesarios, etc.), cómo mantener el difícil 
equilibrio entre novedad (el artículo debe aportar algo original) y tradición (el artículo debe apoyarse en la autoridad de otros trabajos publicados), cómo enfrentarse a las críticas de los revisores anónimos y aprender de ellas, etc.

Finalmente, la socialización científica también supone abrir las puertas de la propia universidad a los visitantes, atrayendo a profesores $\theta$ investigadores, y a estudiantes y becarios de otras universidades. En la misma línea, hay que incluir la capacidad de organizar congresos, simposios y seminarios en la propia universidad. En suma, la capacidad de movilizarse el propio profesor, de atraer a otras personas de fuera que puedan exponer sus puntos de vista, de participar en diversos foros, y de mostrar visibilidad nacional e internacional constituyen aspectos básicos de la socialización académica.

\section{ASUMIR LA CULTURA DE LA EVALUACIÓN}

Algunos podemos recordar un sistema universitario en que los profesores recibían muy pocos recursos para la investigación y la docencia. La "ventaja" de aquellos tiempos un tanto grises es que tampoco se exigía gran cosa al profesor. Éste podía pasar años con un rendimiento profesional mínimo, y nadie parecía notarlo ni echárselo en cara. Con frecuencia, los profesores desarrollaban una labor aislada y provinciana, de escasa repercusión social aparte de la posible influencia que pudieran ejercer sobre sus alumnos. Los proyectos de investigación, las comunicaciones científicas, los intercambios docentes con otras universidades, la movilidad en general, estaban reducidos al mínimo. Por supuesto había una minoría de profesores entusiastas que, a costa de un enorme esfuerzo individual, y buscando recursos fuera de los cauces universitarios, conseguian investigar. Hoy hay muchos más recursos en convocatorias de las propias universidades, regionales, nacionales o europeas e, incluso, en algunos casos se pueden obtener fondos de las empresas privadas. En contrapartida se está instalando entre nosotros la cultura de la evaluación. Tenemos más oportunidades pero también se nos pide más rendimiento. La filosofía de la evaluación es ne- 
cesaria, pues introduce una cierta tensión hacia el logro de metas más ambiciosas que las de la mera rutina diaria, e incentiva los logros. Esta filosofía está implantada desde hace años en todos los sistemas académicos del mundo desarrollado. No es mi intención entrar ahora a juzgar los sistemas de evaluación actualmente vigentes en nuestro país que, en mi opinión, tienen aún graves defectos (burocratización, criterios inadecuados o poco discriminativos, etc.) que deberán corregirse. En cualquier caso el profesor universitario debe tener una actitud abierta y cooperativa ante la evaluación, ya que seguramente ésta no es una moda pasajera, sino un rasgo general del sistema universitario que se irá consolidando y perfeccionando con el tiempo.

\section{SABER ADAPTARSE A UN ENTORNO INSTITUCIONAL POCO IDÓNEO}

Acabo de decir que hay que asumir la cultura de la evaluación. Desgraciadamente las contrapartidas que ofrece la institución universitaria a esta exigencia de evaluación son escasas. No me refiero a los incentivos económicos individuales que reciben los profesores por la producción científica o la docencia, sino al hecho de que la calidad de vida institucional no ha mejorado substancialmente en los últimos años. Los servicios de apoyo a la investigación son deficientes, el personal auxiliar dedicado a estos fines sigue siendo escaso, y su integración en los centros y departamentos disfuncional en comparación con los estándares europeos o norteamericanos. Una consecuencia de ello es que cualquier iniciativa profesional del profesor, como solicitar bolsas de viajes, proyectos de investigación, organizar congresos y seminarios, o desarrollar intercambios Sócrates, le supone asumir directamente tareas administrativas y de gestión adicionales. Por otra parte, la maraña de órganos universitarios (comisiones, consejos de departamento, juntas de facultad, claustros, etc.) le obliga a una constante dispersión de su actividad en múltiples tareas que, aun siendo necesarias algunas de ellas, pueden resultar abrumadoramente distractoras. A todo esto hay que añadir los procesos de cambio institucional que se suceden interminablemente a lo largo de los años: nuevos planes de estudio con 
modificaciones en los contenidos de las materias, en su periodicidad, o en los sistemas de evaluación del alumno; implantación de nuevas titulaciones; cambios en las categorias del profesorado; introducción de nuevos sistemas de oposición; cambios estructurales en los programas de doctorado; nuevas normas sobre la lectura de tesis doctorales, etc. Finalmente, la maquinaria administrativa que rodea la ciencia y la universidad en nuestro país sigue siendo aparatosa y disfuncional. Por ejemplo, los trámites burocráticos en la solicitud y la gestión de proyectos, bolsas de viaje u otras ayudas, pueden eternizarse al incluir controles innecesarios y restricciones que parecen basarse en el principio de la desconfianza hacia el ciudadano.

Un entorno institucional asi puede llegar a ser abiertamente hostil especialmente para los profesores que tienen más iniciativas. Por otra parte, no se me ocurren condiciones ambientales más inapropiadas para la reflexión, la creatividad y la producción intelectual. En un reciente articulo publicado en el Monitor on Psychology de la American Psychological Association (Noviembre, 2003), se describen algunos factores que "matan la creatividad" (creativity killers), tales como la falta de autonomía, la actividad fragmentada por múltiples interrupciones para realizar tareas de bajo nivel, la escasez de recursos, la demanda de metas a muy corto plazo, el exceso de presión temporal, o la reglamentación excesiva del trabajo. Creo que nuestro medio institucional (quizá con algunas excepciones) cumple punto por punto estos factores anti-creatividad.

El profesor universitario en España se encuentra así con desafíos adicionales, difíciles de comprender para sus colegas de otros países. Mi propuesta es que el profesor debe saber "sobrevivir", manteniendo la iniciativa y siendo productivo a pesar de que la institución, anónima y ciega, no parezca estar interesada en ello. Esta respuesta positiva de los profesores más comprometidos a un medio institucional hostil puede tener, además, un valor añadido. Es muy posible que consigan crear "microclimas" institucionales aptos para la formación de investigadores, la mejora de la calidad docente, el desarrollo de proyectos científicos, etc., y todo ello... a pesar de la institución. Naturalmente hay otra forma de sobrevivir en este medio: no tener iniciativas ni complicarse la vida. Pero esta no me parece una respuesta aconsejable. 


\section{CONSIDERACIONES FINALES}

He empezado este escrito con unas reflexiones optimistas acerca del auge y expansión de la psicologia en nuestra sociedad, y he terminado con unas reflexiones bastante amargas sobre el entorno institucional en que nos encontramos muchos profesores universitarios. No obstante, los psicólogos profesionales y los profesores de psicología actuales tienen una preparación comparable a la de otros países próximos. La creciente demanda social de psicólogos en España se explica, sobre todo, por los conocimientos científicos y el saber hacer de nuestros profesionales. Pero ante el inminente cambio en las titulaciones y planes de estudio de psicología -iuna vez más!- hay que estar alerta para que la formación científica del psicólogo no se resienta. Presumiblemente, los nuevos curricula que surjan de la nueva turbulencia institucional, siguiendo las directrices europeas, situarán el énfasis en el fomento de las habilidades profesionales del psicólogo. Esto es muy oportuno, pero en ningún modo debe considerarse incompatible con la formación científica del psicólogo tal como he intentado demostrar en estas páginas. 